EGU2020-12168

https://doi.org/10.5194/egusphere-egu2020-12168

EGU General Assembly 2020

(c) Author(s) 2020. This work is distributed under

the Creative Commons Attribution 4.0 License.

\title{
Accelerated wind conditions: spectral shape evolution and turbulent kinetic energy dissipation from laboratory and field measurements
}

\author{
Lucia Robles-Diaz ${ }^{1}$, Francisco J. Ocampo-Torres ${ }^{2}$, and Hubert Branger ${ }^{3}$ \\ ${ }^{1}$ CICESE, Physical Oceanography Department, Ensenada, Mexico (Irobles@cicese.edu.mx) \\ ${ }^{2}$ CICESE, Physical Oceanography Department, Ensenada, Mexico (ocampo@cicese.mx) \\ ${ }^{3}$ Aix-Marseille Univ, CNRS, Centrale Marseille, IRPHE, Marseille, France (branger@irphe.univ-mrs.fr )
}

\begin{abstract}
A determined shape of the energy wave spectrum can be estimated from a given fetch and wind speed. Also, several studies have characterized the balance of the turbulent kinetic energy under the effect of waves and currents under constant wind conditions. However, deeper research is needed in order to characterize the wind-wave generation processes under non-stationary wind conditions. In this way, to be able to determine the uncertainty on not considering accelerated wind events in the air-sea momentum exchange estimations.
\end{abstract}

Periods of accelerated winds were analyzed from experimental and field data. On one hand, several laboratory experiments were carried out in a large wind-wave facility at the Institut Pytheas (Marseille-France). Momentum fluxes were estimated from hot wire anemometry and, the free surface displacement was measured along the wave tank by resistance and capacitance wire probes. Also, the surface drift current was measured from a profiling acoustic velocimeter. During these experiments, the wind speed goes from $2 \mathrm{~m} / \mathrm{s}$ to reach the maximum wind speed of $13 \mathrm{~m} / \mathrm{s}$. A constant wind acceleration characterizes each test. On the other hand, the field data were obtained from an Oceanographic and Marine Meteorology Buoy (BOMM) located in the Gulf of Mexico, from July 2018 to February 2019. The BOMM was equipped with a sonic anemometer, capacitance wires, and an inertial motion unit. Both sets of data are characterized by a high sampling rate that allows us to directly estimate the wind stress over the sea surface. Also, provide us with useful information about the evolution of the wave spectra and enable us to determine the dissipation rate of turbulent kinetic energy. It was observed that the wind acceleration has a direct effect on the momentum transfer efficiency from the wind to the wave field and that the momentum transfer is reduced as wind acceleration increases. 
